\title{
INSULIN RECEPTORS AND GLUCOSE UPTAKE IN THE HUMAN PARASITE SCHISTOSOMA MANSONI
}

\author{
AHIER A.*, KHAYATH N.*, VICOGNE J.** \& DISSOUS C.*
}

\section{Summary:}

Very little is known about insulin signalling in schistosomes despite its potential importance in host-parasite molecular dialogue and parasite growth and development. The recent characterization of two insulin receptors (SmIR-1 and SmIR-2) in Schistosoma mansoni has led us to reconsider the question of the potential importance of insulin in host-schistosome interactions. In this work, we demonstrated that insulin could regulate glucose uptake in schistosomes and we investigated the implication of SmIR-1 and SmIR-2 in this process. The possibility that specific inhibitors of SmIR-1 and SmIR-2 tyrosine kinase activities could be developed to target schistosomes is discussed.

KEY WORDS : Schistosoma mansoni, insulin receptor, glucose, tyrosine kinase inhibitor.

\section{INTRODUCTION}

$\circlearrowleft$ chistosomiasis or bilharziasis is a water-borne disease caused by a parasitic flatworm of the genus Schistosoma. The disease affects several hundred million people in 75 tropical and sub-tropical countries leading to 280,000 deaths annually in subSaharan Africa alone (Gryseels et al., 2006). Moreover, recent studies indicated that the impact of schistosomiasis on the global burden of disease has been seriously underestimated (King et al., 2006).

Schistosomes have a complex life cycle, with different parasite stages perfectly adapted to develop in their hosts and to assure active propagation and transmission. In the mollusc intermediate host, sporocysts multiply intensively and generate huge numbers of cercariae infective for the vertebrate definitive host. Similarly, sexually mature worms developing in the vertebrate host produce large numbers of embryonic eggs that will assure an intense level of transmission of this

\footnotetext{
* Inserm U547, Lille, Université Lille 2, Institut Pasteur de Lille, IFR142, Lille, France.

** UMR8161-CNRS, Institut de Biologie de Lille, France.

Correspondence: Dr Colette Dissous, U547 Inserm, Institut Pasteur, 1, rue du Professeur A. Calmette, 59019 Lille Cedex France.

Tel.: 33 (0)32087 7350 - Fax: 33 (0)3 20877888 .

E-mail: colette.dissous@pasteur-lille.fr
}

Résumé : RÉCEPTEURS DE L'INSULINE ET PRISE DE GLUCOSE CHEZ LE PARASITE HUMAIN SCHISTOSOMA MANSONI

Très peu de choses sont connues concernant la signalisation insuline chez le schistosome malgré son importance potentielle dans le dialogue hôte-parasite ainsi que dans la croissance et le développement du parasite. La caractérisation récente de deux récepteurs à l'insuline chez Schistosoma mansoni (SmIR-1 and SmIR-2) nous a conduit à reconsidérer la question de l'importance potentielle de l'insuline dans les interactions hôte-schistosome. Dans ce travail, nous avons démontré que l'insuline peut réguler la prise de glucose chez le schistosome et nous avons évalué l'implication de SmIR-1 et de SmIR-2 dans ce processus. La possibilité de développer des inhibiteurs spécifiques de l'activité tyrosine kinase de SmIR-1 et SmIR-2 pour cibler le schistosome est discutée.

MOTS CLÉS : Schistosoma mansoni, insuline, récepteur, glucose, inhibiteur, tyrosine kinase.

water-borne disease. Intense egg laying by female worms is also responsible for the severe pathologies observed in schistosomiasis, in which trapping of eggs into host tissues leads to the formation of inflammatory granulomas particularly deleterious for hepatic organs. Such processes of parasite multiplication and proliferation are highly energy consuming, depending essentially on host nutritional resources.

Nutritional dependence on host glucose has been well documented for schistosomes. Adult worms import huge amounts of blood glucose from portal and mesenteric veins and can consume every $5 \mathrm{~h}$ their dry weight of glucose (Bueding, 1950). Glucose is mainly taken up across the worm tegument by facilitated diffusion, involving two glucose transporters SGTP1 and SGTP4 asymmetrically distributed on the basal and apical membranes of the schistosome tegument (Skelly et al., 1994). Whereas SGTP1 is found only within the basal membrane, SGTP 4 is present within the apical membrane and its expression at the parasite surface seems to be tightly regulated and concomitant with the appearance of the apical membrane bilayer that is formed soon after invasion of cercariae in the host. SGTP4 was shown to be maintained at the surface of all intramammalian stages, suggesting its crucial importance for sugar uptake from host bloodstream and therefore parasite nutrition (Skelly \& Shoemaker, 1996). 
However, the precise mechanisms that could regulate its expression as well as its precise functions in the control of glucose entry in schistosomes still remain poorly known. In mammals, glycemia is regulated by insulin that activates an insulin receptor tyrosine kinasedependent pathway and provokes glucose entry in muscle and fat cells via the translocation of GLUT4 (glucose transporter 4) molecules to the plasmatic membrane (Khan \& Pessin, 1996). The recent characterization in our laboratory of two distinct $S$. mansoni insulin receptor homologs SmIR-1 and SmIR-2, and the demonstration of the capacity of their ligand binding domains to bind specifically human pro-insulin in yeast two-hydrid assays (Khayath et al., 2007), led us to reconsider the question of the potential importance of insulin in host-schistosome interactions. In this work, we studied the effect of insulin on glucose uptake in schistosomes and we investigated the implication of SmIR-1 and SmIR-2 in this process. Structural analysis of parasite receptor kinase domains was performed in order to investigate the possibilities to develop specific inhibitors of SmIR-1 and SmIR-2 tyrosine kinase activities.

\section{MATERIALS AND METHODS}

\section{PARASITE MATERIAL}

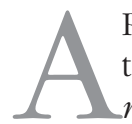

Puerto-Rican strain of $S$. mansoni was maintained by passage through albino Biomphalaria glabrata snails and Mesocricetus auratus hamsters. Adult schistosomes were collected by portal perfusion from infected hamsters. Schistosomula were prepared as previously described (Dissous \& Capron, 1981).

\section{DRUG TREATMENT AND GLUCOSE UPTAKE ASSAYS}

Parasites (10 adult worm pairs or 3,000 schistosomula) were incubated in $250 \mu \mathrm{L}$ of DMEM (Gibco) supplemented by $2.5 \mathrm{mM}$ glucose, without (control) or with 0.1 and $1 \mathrm{mM}$ bovine insulin final concentrations (Sigma) for a $30 \mathrm{~min}$ period. Glucose uptake was then measured by adding $1 \mu \mathrm{Ci}$ of [ $\left.{ }^{3} \mathrm{H}\right] 2$-DOG (specific activity $12 \mathrm{Ci}$ / mmol, Amersham) for five min. After extensive washing in DMEM, parasites were solubilized in $200 \mu \mathrm{L}$ of $0.15 \mathrm{M} \mathrm{NaOH}$ and the amount of radioactivity was determined in each lysate. Similar experiments were performed in which parasites were incubated in DMEM supplemented by $2.5 \mathrm{mM}$ glucose and by $5 \%$ fetal bovine serum (as a source of insulin), without or with tyrphostin AG1024 (Sigma), tyrphostin AG538 (Sigma) or sodium orthovanadate (Sigma) for $30 \mathrm{~min}$. Levels of glucose incorporation were calculated in cpm per $\mathrm{mg}$ of protein in each sample and expressed for each experimental condition as the percentage of the value found in respective control.

\section{RNA INTERFERENCE EXPERIMENTS}

dsRNAs of SmIR-1 and SmIR-2 were synthesized from cDNA encoding $S$. mansoni insulin receptors (Genbank $\mathrm{n}^{\circ}$ AF314754 and AY649844 respectively) using genetargeted primers containing T7 promoter sequences. dsRNAs were synthesized and purified using the Megascript $^{\mathrm{TM}} \mathrm{T} 7 \mathrm{kit}$ (Ambion) according to the manufacturer's instructions. Newly-transformed schistosomula were incubated in M199 medium (Gibco) during six days with $350 \mu \mathrm{g} / \mathrm{mL}$ of SmIR-1 dsRNA (positions 181-1134) or SmIR-2 dsRNA (positions 733-1648) using irrelevant SmFtz-F1 dsRNA (Genbank n AY028787, positions 1310-1746) as control, and then analyzed for their capacity to incorporate $[3 \mathrm{H}] 2-\mathrm{DOG}(5 \mu \mathrm{Ci} / \mathrm{mL})$ during a five min period.

\section{Molecular modelling OF ATP-BINDING SITES}

The hIR tyrosine kinase domain was submitted to the automated homology modelling server (PS)2 (bttp:// ps2.life.nctu.edu.tw/) and hIR protein database (PDB) entry 1IR3 was chosen as template. Three-dimensional structures of SmIR-1 and SmIR-2 were built using RAMP (bttp://www. ram.org/ computing/ramp/) and visualized using Accelrys DS Visualizer V1.7 (Accelrys Software Inc ${ }^{\mathcal{O}}$ ). Amino acid sequences of hIR (position 1002-1149), SmIR-1(position 1196-1372) and SmIR-2 (position 1171-1430) ATP-binding domains were aligned by the CLUSTAL W method as described previously (Khayath et al., 2007).

\section{RESULTS AND DISCUSSION}

$\mathrm{D}$ ifferent studies have demonstrated that the development of $S$. mansoni could be dependent on host hormones (De Mendonca et al., 2000) and growth factors (Dissous et al., 2006) but the possibility that insulin-dependent mechanisms also occur in schistosomes has been extensively debated. Indeed, the first studies showing that insulin could increase oxygen and glucose uptake, life span and resistance to antibodies of schistosomula (Levi-Schaffer \& Smolarsky, 1981) have been further disapproved by other authors who concluded to an insulin independence of schistosomes (Clemens \& Basch, 1989).

As a preliminary step to this study, we decided to analyze the effect of a purified fraction of human insulin on glucose entry in in vitro cultured adult worms and schistosomula using tritiated 2-deoxyglucose $\left(\left[{ }^{3} \mathrm{H}\right] 2\right.$ 2DOG) as a radioactive indicator. Results in Figure 1 show that a $30 \mathrm{~min}$ incubation with $1 \mathrm{mM}$ insulin (a dose of insulin currently used to stimulate mammalian cells 
A-

Glucose uptake (\% of control)

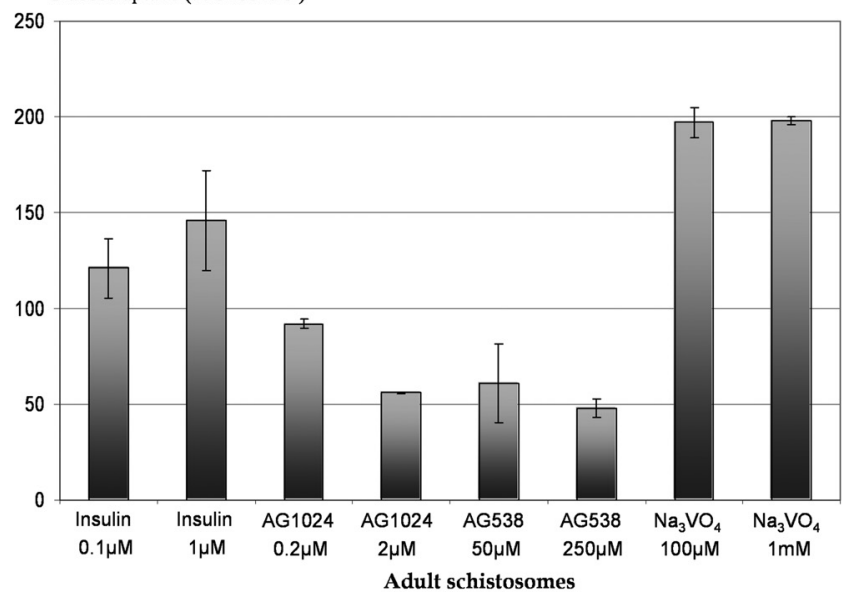

B-

Glucose uptake (\% of control)

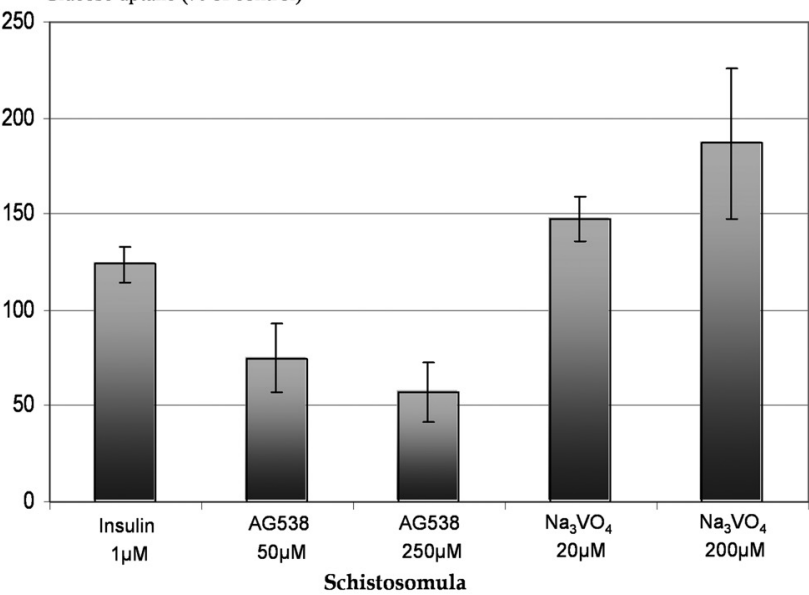

Fig. 1. - Regulation of glucose uptake by insulin-dependent kinase pathways in parasites.

S. mansoni adult schistosomes (A) or schistosomula (B) were incubated with insulin, tyrphostin AG1024 or AG538 or sodium orthovanadate. Levels of $\left[{ }^{3} \mathrm{H}\right] 2-\mathrm{DOG}$ incorporation were calculated in cpm per $\mathrm{mg}$ of protein in each sample and expressed as percentages of the value found in untreated control parasites (mean of three independent experiments $+/$ - standard deviation).

(Caraiscos et al., 2007)), can induce an increase of the quantity of $\left[{ }^{3} \mathrm{H}\right] 2-D O G$ contained in adult worms ( $\sim 150 \%$ of control) and in schistosomula ( 125\% of control), therefore indicating that insulin dependence could exist in these $S$. mansoni stages and suggesting that SmIR-1 and SmIR-2 might be involved in signalling at the host-parasite interface.

Two insulin receptors SmIR-1 (Genbank n AF314754) and SmIR-2 (Genbank n ${ }^{\circ}$ AY649844) have been recently characterized in S. mansoni and shown to differ by several structural motifs known to be essential for signalling, as well as by their site of expression in the parasite. These data constituted the first report of a diversification of IR family in invertebrates (Khayath et al., 2007). One possible hypothesis about this diversification was that SmIR-1 and SmIR-2 could have distinct and specialized functions, as in mammals in which insulin receptors (INR) and insulin like growth factor-1 receptors (IGR) are specialized in the regulation of glycemia and growth control respectively (Kim \& Accili, 2002). Interestingly, SmIR-1, but not SmIR-2, was localized in the $S$. mansoni tegument together with the glucose transporters SGTP1 and SGTP4, suggesting a potential implication of SmIR-1 in the regulation of glucose uptake in $S$. mansoni, similar to that described for mammal INRs. At the opposite, the diffuse expression of SmIR-2 observed in the parasite parenchyma could argue for its possible function in growth control as in the case of mammal IGRs. In recent years, RNA interference has been proved to be a potent approach for loss-of-function studies in schistosomes, particularly for several enzyme activities associated with the tegument (Ndegwa et al., 2007). In order to evaluate the role of SmIR molecules in glucose uptake, we performed RNAi experiments in S. mansoni schistosomula using the protocol of soaking described by Skelly et al. (2003).

In these conditions, results showed a significative decrease (about $33 \%$ ) of $\left[{ }^{3} \mathrm{H}\right]$ 2-DOG uptake in schistosomula incubated with SmIR-1 dsRNA as compared to parasites incubated with irrelevant SmFtz-F1 dsRNA whereas incorporation of labelled glucose in schistosomula treated by SmIR-2 dsRNA was similar to that found in control parasites. However, if such results were consistent with a possible implication of SmIR-1 in the regulation of glucose uptake in parasites, they still remain to be confirmed. Indeed, for unknown reasons, we were not able to correlate the decrease of glucose uptake in the dsRNA-treated parasites to a significant extinction of SmIR-1 transcripts. One possible explanation could be that the soaking technique used in these assays promotes a preferential addressing of dsRNA into the tegument, and therefore the selective targeting of SmIR-1 transcripts present in the syncitium without affecting the global level of parasite SmIR-1 transcripts also expressed in muscles and intestinal epithelium, as previously shown (Khayath et al., 2007). Additional experiments in which electroporation was used for dsRNA delivery (Ndegwa et al., 2007) did not result in a more efficient suppression of transcripts. In order to pursue our investigations on a potential role of insulin receptor signalling in schistosomes, we analyzed the effect of molecular compounds, known to modulate specifically the activity of the insulin receptor, on the capacity of parasites to incorporate radiolabelled deoxyglucose. Two tyrosine kinase (TK) inhibitors (tyrphostins AG1024 (Parrizas et al., 1997) and AG538 (Blum et al., 2000) were tested in these experiments using concentrations roughly similar to those already shown to inhibit efficiently INR and IGR on different 
human cell lines (Guha et al., 2007; Blum et al., 2003). Results in Figure 1A indicate a significant decrease $(45 \% \pm 5)$ of radioactive glucose in in vitro-cultured adult worms treated with $2 \mu \mathrm{M}$ AG1024 as well as in

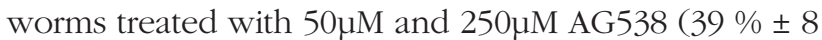
and $52 \% \pm 2$ respectively). A marked inhibitory effect of AG538 was also observed in schistosomula larvae incubated with $50 \mu \mathrm{M}$ or $250 \mu \mathrm{M}$ AG538 (25\% \pm 17 or $43 \% \pm 15$ reduction in glucose uptake, respectively) (Fig.1B). Inversely, the addition of $\mathrm{Na}_{3} \mathrm{VO}_{4}, \mathrm{a}$ potent phosphatase inhibitor, induced a marked increase of the quantity of labelled glucose recovered both in adult worms and schistosomula (Fig. 1A, B), suggesting that glucose uptake in intramammalian stages of S. mansoni was dependent on phosphorylation processes and could be regulated by insulin-dependent pathways, which have to be further studied. Indeed, if it seems very likely that the mechanism of glucose uptake implies, as in mammals (Khan \& Pessin, 2002), modulation of glucose transporter activities, we cannot exclude the participation of the schistosome hexokinase activity in this process (Tielens et al., 1994). Since this enzyme catalyses the first irreversible step of glucose uptake by phosphorylating glucose molecules and thereby prevents their exit from the cell, it could also be in part responsible for the increase of glucose uptake in the parasites.

In spite of all efforts made for reducing schistosome transmission, particularly by improving water supplies, sanitation conditions and snail host population control in endemic areas, mass treatment of human populations by chemotherapy still remains the most efficient tool to control morbidity and mortality due to schistosomiasis (Fenwick \& Webster, 2006). Since the mid1980s praziquantel (PZQ) has been the drug of choice for bilharziasis and although it is less effective in killing immature worms than mature worms, PZQ is still the only choice available. However, prolonged and intensive use of $\mathrm{PZQ}$ in monotherapy raises serious concerns about development of resistance to this drug and several cases of tolerance/resistance to PZQ have already been reported. It is therefore essential to discover novel molecular targets in order to develop new chemical drugs applicable to anti-schistosome therapy (Caffrey, 2007).

During the last two decades, the importance of glucose as a major and essential nutrient for intramammalian stages of $S$. mansoni has motivated much efforts to characterize parasite proteins involved in its transport and metabolism and to evaluate the potential interest of such molecules in novel immunological or pharmacological strategies for control of schistosomiasis. In this context, the schistosome glucose transporters SGTP1 and SGTP4 were first considered as potential efficient targets for a reduction of glucose consumption in adult worms (Skelly \& Shoemaker,
1996), but the molecules that could regulate their function and their expression at the parasite surface could be similarly considered as putative targets for new drugs. SmIR-1 and SmIR-2 insulin receptors with kinase functions which are likely able to modulate glucose in parasites could thus represent potential candidates.

Protein tyrosine kinases (PTK) constitute important therapeutic targets in a number of cancers and the development of Tyrphostins (TYRosine PHOSphorylation INhibitors) has greatly modified the approach to cancer therapy. Aberrations in PTK signalling are also responsible for metabolic disorders in diabetes and inflammatory diseases. Tyrphostins were derived from natural and poorly selective compounds (genistein, lavendustin A) serving as lead compounds to develop laboratory-synthesized tyrphostins which were shown to be more potent and selective. Initial tyrphostins were hydroxyphenyl-containing molecules mimicking tyrosine and thus active as substrate competitors. Then the incorporation of an atomic ring to these small molecules led to the generation of bicyclic tyrphostins (anilinoquinazolines, anilinoquinolines and anilinopyridopyrimidines) found to be ATP competitive or mixed competitive. Currently, most of the available PTK inhibitors are reversible ATP mimics that require high doses to compete with the high levels of endogenous ATP. For this reason, efforts are made to develop more potent irreversible ATP mimics as well as more selective substrate mimics, targeting a substrate-domain less conserved than the ATP-binding site (Levitzki \& Gazit, 1995).

Although ATP-binding sites are highly conserved among tyrosine kinases (Moretti et al., 2006; Vulpetti \& Bosotti, 2004), it has been shown that minor differences in kinase domain structures can lead to the development of selective inhibitors able to recognize specific PTKs. According to this, it seems very likely that any structural divergences between catalytic domains of host and schistosome PTKs should be exploited for the design of molecules able to target schistosome PTKs without affecting human host kinases (Dissous et al., 2007).

Our results showing that two inhibitors of mammal IR kinase activity (Tyrphostins AG1024 and AG538) could potentially inhibit the activity of SmIR-1 and SmIR-2 molecules already suggested conservation between parasite and mammal receptors. Comparative threedimensional modeling of schistosome (SmIR-1 and SmIR-2) and human (hIR) insulin receptors confirmed the structural conservation of the ATP-binding site of SmIR-1 and SmIR-2 (Fig. 2). Indeed, the consensus

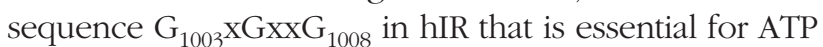
binding is conserved in SmIR-1 and SmIR-2 as well as the consensus $\mathrm{V}_{1027} \mathrm{AVK}$ motif also implicated in ATP binding and necessary for its stabilization in the catalytic pocket. However, in these consensus sequences, 


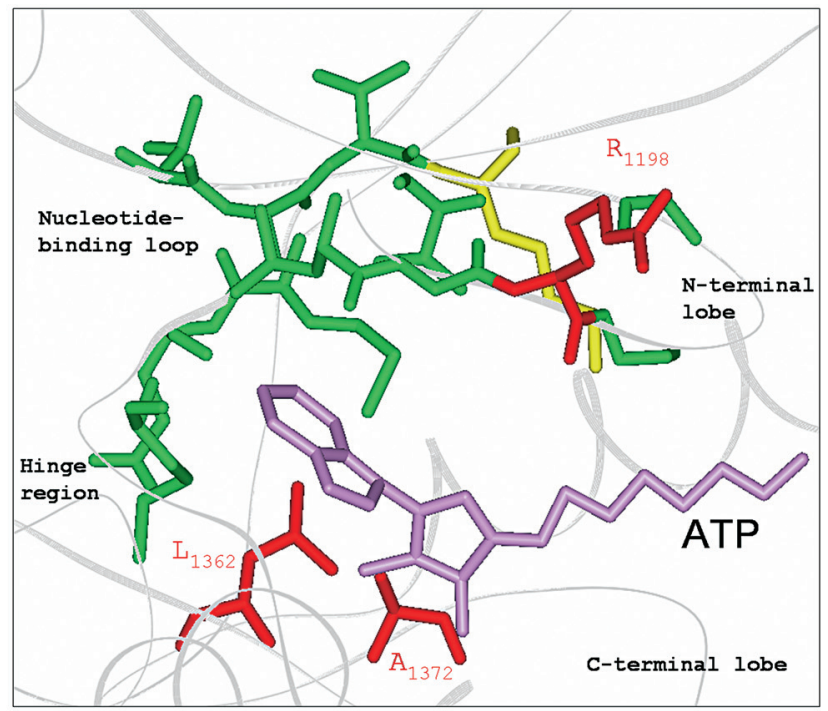

SmIR-1 ATP binding domain

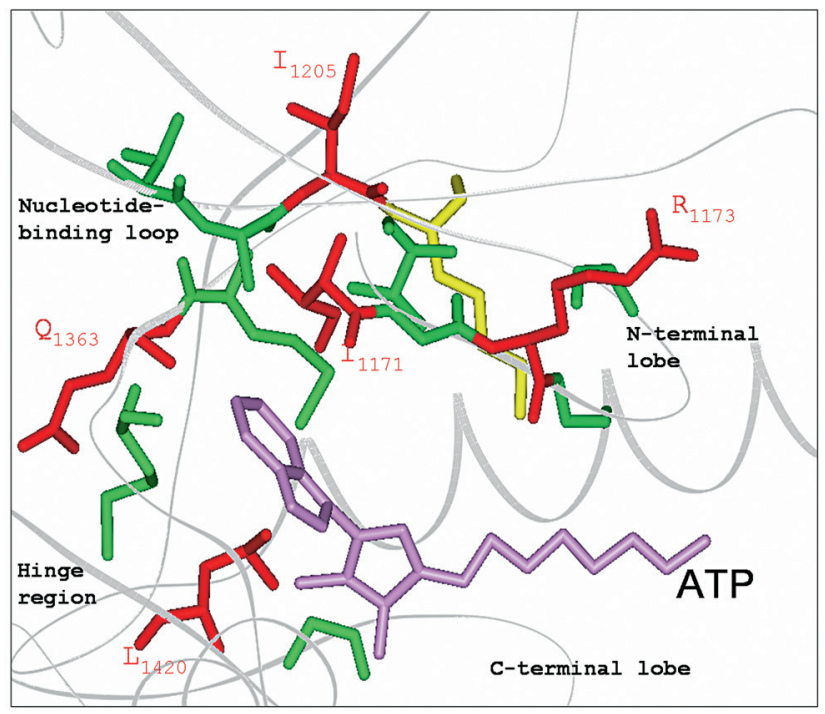

SmIR-2 ATP binding domain

\section{B}

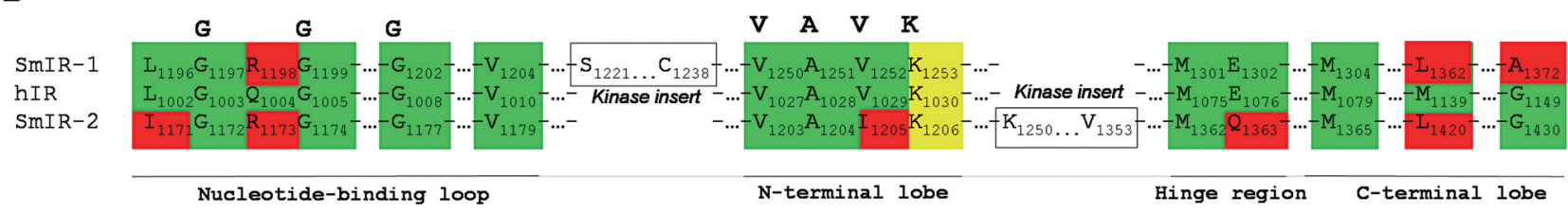

Fig. 2. - Molecular modelling of ATP-binding pockets from SmIR-1 and SmIR-2 receptors (A) and alignment of residues with human hIR sequence (B).

The different domains surrounding ATP- binding cleft are defined according to Moretti et al., 2006. Lysine residues susceptible to bind ATP are in yellow. Conserved residues constituting the ATP-binding sites of SmIR-1, SmIR-2 and hIR are in green. In red are residues not conserved in SmIR-1 or SmIR-2 as compared to hIR.

$\mathrm{Q}_{1004}$ in hIR is replaced by arginine residues both in SmIR-1 and SmIR-2 ( $\mathrm{R}_{1173}$ and $\mathrm{R}_{1198}$ respectively) and $\mathrm{V}_{1027}$ of hIR by $\mathrm{I}_{1205}$ in SmIR-2. These residues are not known to interact directly with ATP but they are involved in the conformation of the ATP binding domain. Other important residues involved in ATP binding have been described (Moretti et al., 2006, Vulpetti \& Bosotti, 2004). They correspond in hIR to $\mathrm{L}_{1002}$ and $\mathrm{V}_{1010}$ (from the nucleotide binding loop), $\mathrm{A}_{1028}$ (from the core of the N-terminal lobe), $\mathrm{M}_{1139}$ and $\mathrm{M}_{1079}$ (from the C-terminal lobe) and they bind to the adenine pyrimidine ring of the ATP molecule defined as the ligand core. Figure 2 shows that these essential residues are conserved in SmIR-1 and SmIR-2 with expected variability $\left(\mathrm{L}_{1002}\right.$ replaced by $\mathrm{I}_{1171}$ in SmIR-1 and $\mathrm{M}_{1139}$ by leucine residues in SmIR-1 and SmIR-2), indicating that the cleft representing the ATP-binding site is perfectly conserved in parasite receptors and contains all the residues involved in ligand core binding.

However, further comparison of SmIR-1, SmIR-2 and hIR sequences also highlighted important differences between parasite and human kinase domains that could be potentially exploited in the design of specific inhibitors. The specificity of ATP mimic inhibitors has been shown to be mainly determined by the presence of differential residues close to the ATP binding site (Levitzki \& Gazit, 1995). In this context, we could note that several residues present in the ATP-binding cleft of hIR are not conserved in SmIR-1 and SmIR-2. For example, $Q_{1004}$ in hIR is replaced by $R_{1198}$ and $R_{1173}$ in SmIR-1 and SmIR-2 respectively. $G_{1149}$ in hIR is substituted by $\mathrm{A}_{1372}$ in SmIR-1 and $\mathrm{V}_{1029}$ and $\mathrm{E}_{1076}$ in hIR by $\mathrm{I}_{1205}$ and $\mathrm{Q}_{1363}$ in SmIR-2. Moreover, we detected the presence of a large insert (103 residues) in the hinge region of the SmIR-2 kinase domain as well as a smaller sequence (17 residues) inserted in the ATPbinding site of SmIR-1. The existence of these kinase inserts could be considered as schistosome RTK signatures, possibly helpful for the design of inhibitors.

Although little is known about signal transduction pathways in schistosomes, recent genome and transcriptome analyses have given evidence that signalling mechanisms which are essential for the development of metazoans, are mostly conserved in these platyhelminth parasites, and could be involved in the regulation of their complex life-cycle and the interactions with the hosts. In this report, we propose that tyro- 
sine kinase activities of SmIR-1 and SmIR-2 molecules could represent novel potential targets for emerging therapies against schistosomiasis, as it was also suggested for the soluble Src-like kinase of $S$. mansoni (TK3) involved in parasite reproductive activity (Knobloch et al., 2006). Taking into account the recent progress made in the characterization of other platyhelminth tyrosine kinases and particularly the demonstration of the importance of insulin signalling in larval cestode development (Brehm et al., 2006), we can speculate that common strategies based on parasite TK inhibition could be helpful to combat in future diverse phylogenetically related platyhelminths including cestode and trematode species.

\section{ACKNOWLEDGEMENTS}

$\checkmark$ his work was supported by the Institut National de la Santé et de la Recherche Médicale (INSERMFIOCRUZ program), Institut Pasteur de Lille and by the Ministère de l'Éducation Nationale et de la Recherche. The authors thank J. Trolet for his excellent technical assistance.

\section{REFERENCES}

Blum G., Gazit A. \& Levitzki A. Substrate competitive inhibitors of IGF-1 receptor kinase. Biochemistry, 2000, 39, 15705-15712.

Brehm K., Spiliotis M., Zavala-Gongora R., Konrad C. \& Frosch M. The molecular mechanisms of larval cestode development: first steps into an unknown world. Parasitology International, 2006, 55 (Suppl.), S15-S21.

Brichard S.M., Assimacopoulos-Jeannet F. \& Jeanrenaud B. Vanadate treatment markedly increases glucose utilization in muscle of insulin-resistant $\mathrm{fa} / \mathrm{fa}$ rats without modifying glucose transporter expression. Endocrinology, 1992, 131, 311-317.

BuEDING E. Carbohydrate metabolism of Schistosoma mansoni. The Journal of General Physiology, 1950, 33, 475-495.

CAFFrEY C.R. Chemotherapy of schistosomiasis: present and future. Current Opinion in Chemical Biology, 2007, 11, 433-439.

Caraiscos V.B., Bonin R.P., Newell J.G., Czerwinska E., MacDONALD J.F. \& ORSER B.A. Insulin increases the potency of glycine at ionotropic glycine receptors. Molecular Pharmacology, 2007, 71, 1277-1287.

Clemens L.E. \& BASCH P.F. Schistosoma mansoni: insulin independence. Experimental Parasitology, 1999, 68, 223229.

De Mendonca R.L., Escriva H., Bouton D., Laudet V. \& PierCe R.J. Hormones and nuclear receptors in schistosome development. Parasitology Today, 2000, 16, 233-240.

Dissous C. \& CAPRON A. Isolation and characterization of surface antigens from Schistosoma mansoni schistosomula. Molecular and Biochemical Parasitology, 1981, 3, 215-225.
Dissous C., Khayath N., Vicogne J. \& Capron M. Growth factor receptors in helminth parasites: signalling and hostparasite relationships. FEBS Letter, 2006, 580, 2968-2975.

Dissous C., Ahier A. \& Khayath N. Protein tyrosine kinases as new potential targets against human schistosomiasis. Bioessays, 2007, 29, 1281-1288.

FENwick A. \& Webster J.P. Schistosomiasis: challenges for control, treatment and drug resistance. Current Opinion in Infectious Diseases, 2006, 19, 577-582.

Gryseels B., Polman K., Clerinx J. \& Kestens L. Human schistosomiasis. Lancet, 2006, 368, 1106-1118.

Kapp K., Knobloch J., Schussler P., Sroka S., Lammers R., Kunz W. \& Grevelding C.G. The Schistosoma mansoni Src kinase TK3 is expressed in the gonads and likely involved in cytoskeletal organization. Molecular and Biochemical Parasitology, 2004, 138, 171-182.

KHAN A.H. \& Pessin J.E. Insulin regulation of glucose uptake: a complex interplay of intracellular signalling pathways. Diabetologia, 2002, 45, 1475-1483.

Khayath N., Vicogne J., Ahier A., BenYounes A., Konrad C., Trolet J., Viscogliosi E., Brehm K. \& Dissous C. Diversification of the insulin receptor family in the helminth parasite Schistosoma mansoni. Febs Journal, 2007, 274, 659676.

KIM J.J. \& AcCILI D. Signalling through IGF-I and insulin receptors: where is the specificity? Growth Horm E IGF Research, 2002, 12, 84-90.

King C.H., STurrock R.F., Kariuki H.C. \& Hamburger J. Transmission control for schistosomiasis - why it matters now. Trends in Parasitology, 2006, 22, 575-582.

Knobloch J., Kunz W. \& Grevelding C.G. Herbimycin A suppresses mitotic activity and egg production of female Schistosoma mansoni. International Journal for Parasitology, 2006, 36, 1261-1272.

LEVI-SCHAFFER F. \& SMOLARSKY M. Schistosoma mansoni: effect of insulin and a low-molecular-weight fraction of serum on schistosomula in chemically defined media. Experimental Parasitology, 1981, 52, 378-385.

LevitzKi A. \& GaziT A. Tyrosine kinase inhibition: an approach to drug development. Science, 1995, 267, 1782-1788.

Moretti L., TChernin L. \& Scapozza L. Tyrosine kinase drug discovery: what can be learned from solved crystal structures? ARKIVOC VIII, 2006, 38-49.

Ndegwa D., Krautz-Peterson G. \& Skelly P.J. Protocols for gene silencing in schistosomes. Experimental Parasitology, 2007, 117, 284-291.

Parrizas M., Gazit A., Levitzki A., Wertheimer E. \& LeRoith D. Specific inhibition of insulin-like growth factor-1 and insulin receptor tyrosine kinase activity and biological function by tyrphostins. Endocrinology, 1997, 138, 1427-1433.

Skelly P.J., Kim J.W., Cunningham J. \& Shoemaker C.B. Cloning, characterization, and functional expression of cDNAs encoding glucose transporter proteins from the human parasite Schistosoma mansoni. Journal of Biological Chemistry, 1994, 269, 4247-4253.

Skelly P.J. \& Shoemaker C.B. Rapid appearance and asymmetric distribution of glucose transporter SGTP4 at the 
apical surface of intramammalian-stage Schistosoma mansoni. Proceedings of the National Academy of Sciences, USA, 1996, 93, 3642-3646.

Skelly P.J., DA'Dara A. \& Harn D.A. Suppression of cathepsin B expression in Schistosoma mansoni by RNA interference. International Journal for Parasitology, 2003, 33, 363-369.

Vicogne J., Cailliau K., Tulasne D., Browaeys E., Yan Y.T., Fafeur V., Vilain J.P., Legrand D., Trolet J. \& Dissous C. Conservation of epidermal growth factor receptor function in the human parasitic helminth Schistosoma mansoni. Journal of Biological Chemistry, 2004, 279, 37407-37414.

Vulpetti A. \& BosotTi R. Sequence and structural analysis of kinase ATP pocket residues. Farmaco, 2004, 59, 759-765.

Reçu le 21 mai 2008 Accepté le 24 juillet 2008 